\title{
The perceptual development of a British-English phoneme contrast in Dutch adults
}

\author{
Willemijn Heeren \\ Utrecht University
}

\begin{abstract}
How does the perception of a new phoneme contrast develop? In answering this question we consider two hypotheses: i) Acquired Distinctiveness: before learning, differences between and within phoneme categories are hardly discriminable. Through training, the phoneme boundary is learnt. ii) Acquired Similarity: before learning, differences between and within phoneme categories are well discriminated. Through training, only the phoneme boundary remains discriminable. In a pretest-training-posttest design, Dutch adults learnt the BritishEnglish pseudowords thif and sif: the first consonant in thif is not a phoneme of Dutch. Between pretest and posttest with materials from one speaker, participants were trained with speech from five other speakers. This forced listeners to form abstract phoneme categories. The results show that trained listeners performed better in the posttest than control listeners. However, in general the control group, who received no training, was difficult to distinguish from the trained listeners. With respect to the research question we found that discrimination levels increased as a result of training.
\end{abstract}

\section{Introduction}

The world's languages differ in their phoneme inventories. While learning a first or second language, a listener must acquire such a set of phonemes. In the continuous flow of speech sounds, however, two instances of one phoneme may show great diversity, due, for example, to speaker characteristics or speech rate. Moreover, two acoustically similar speech sounds may actually be realizations of two different phonemes. Native listeners tend to put sharp boundaries between phoneme categories in their language. Generally, discrimination of sounds taken from different sides of the phoneme boundary is higher than discrimination of within-category tokens. Assuming that this discrimination pattern is a native listener's end state, this would also be what a language learner is trying to achieve. But how does one learn to perceive novel phonemes?

Early studies that tried to change phoneme perception through laboratory training were not very successful (see Strange \& Jenkins, 1978 for a review). Later studies, however, have shown that nonnative phoneme contrasts can be learnt through relatively short laboratory training (e.g. Jamieson \& Morosan, 1986; Lively, Logan, \& Pisoni, 1993). But there are also nonnative contrasts for which training is unnecessary. Best, Traill, Carter, Harrison, \& Faber, (2003) showed that nonnative phoneme contrasts that do not fall within the acoustic range exploited by one's native language, can be discriminated quite well.

To study the perceptual development of novel phoneme contrasts, we consider two opposing hypotheses, Acquired Distinctiveness and Acquired Similarity, that have been proposed to explain the learning of native phonemes (Liberman, Harris, Kinney, \& Lane, 1961). Both hypotheses deal with the degree to which within-category and between-category differences can be discriminated by a listener. The first hypothesis, Acquired Distinctiveness, says that listeners learn to perceive differences between those speech sounds that they are trained to 
categorize differently, although they were unable to hear any differences before learning this new phoneme contrast. After training, discrimination of stimuli on different sides of the phoneme boundary has improved. In support of this hypothesis, a training study by Jamieson and Morosan (1986) reported increased discrimination at the phoneme boundary without within-category improvement.

The second hypothesis, Acquired Similarity, states that both within-category and betweencategory speech sounds can be distinguished well before training. As a result of training, however, perceptual sensitivity to speech sounds that belong to the same category decreases, in such a way that only above-chance discrimination of the stimuli straddling the phoneme boundary remains. This type of learning seems similar to the way infants treat speech sounds during their first year of life (Pisoni, 1991). Werker \& Tees (1984), for example, have shown that infants of six to eight months of age can discriminate a natural speech contrast that their language environment does not contain, and that is not discriminated by adults from that language. But after about ten to twelve months infants lose the ability to discriminate these phoneme pairs. It is not probable, however, that the infants' representations lie at a phonemic level.

The question this paper aims to answer is: how do Dutch listeners learn the British English $/ \theta-\mathrm{s} /$ contrast, where $/ \theta /$ is not a phoneme of Dutch? Do Dutch adults learn this contrast in accordance with Acquired Distinctiveness or Acquired Similarity? We tried to answer this question by means of a laboratory training study, run with Dutch adult listeners. Both before and after training, the perception of the nonnative phoneme continuum was assessed in absolute identification and discrimination tests. A control group that did not participate in training sessions, was also tested.

A subquestion that was addressed in the present study is whether perceptual learning is influenced by whether or not subjects know which language they are learning. Since most Dutch have learnt some English in primary and secondary school, the $/ \theta$-s/ contrast may not be entirely new to them. However, knowing that $/ \theta /$ is different from $/ \mathrm{s} /$ does not imply that a Dutch listener can differentiate the two acoustically. Moreover, the Dutch often produce /s/ when trying to pronounce $/ \theta$ / (Collins \& Mees, 1999), which shows that they have difficulties with the English phoneme. We wanted to find out whether listeners who are told that they are learning a contrast from English, a language they are not completely unfamiliar with, benefit from this knowledge as opposed to learners who are told that they are learning a phoneme contrast from a foreign language.

\section{Method}

\subsection{Materials}

Eight-step continua were synthesized for the British-English phoneme contrast $/ \theta$-s/ by means of linear spectral interpolation (van Hessen, 1992). The phonemes occurred in the onset position of a pair of (both in Dutch and in English) nonsense words: thif $\sim$ sif. Nonsense words were used to exclude word frequency effects or lexical bias (cf. Ganong, 1980). The phoneme continua were based on speech from six speakers of Standard English, both males and females. Figure 1 shows spectrograms of the eight-step continuum from one of the six speakers. The location of the phoneme boundary in each continuum had been determined from a classification study with 31 native British English listeners. ${ }^{1}$

\footnotetext{
${ }^{1}$ Collection of the materials was supported by grant R30-579 from the Netherlands Organisation for Scientific Research (NWO).
} 


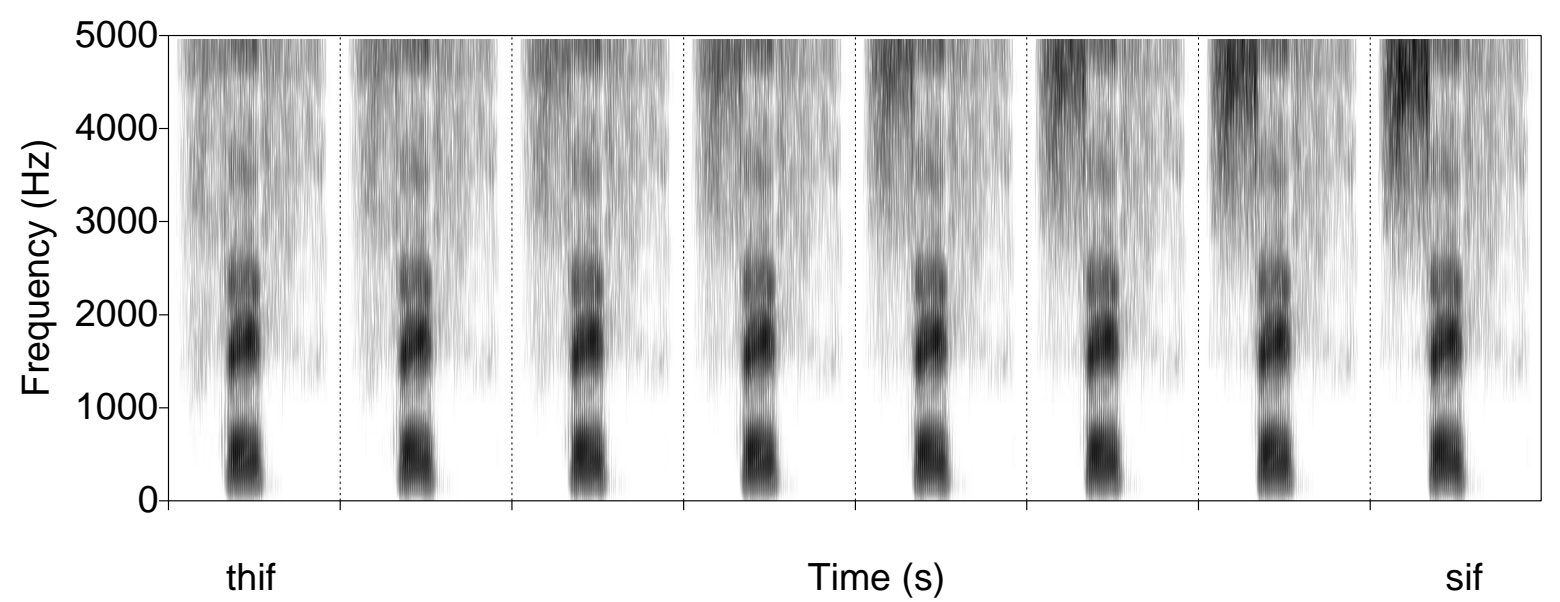

Figure 1. Spectrograms of the thif-sif continuum from one of the speakers.

\subsection{Participants}

Thirty-four students, native speakers of Dutch reporting normal hearing, participated in the test. None of them were or had been students of English. Seventeen subjects received training between pretest and posttest. The other half, the control group, participated only in pretest and posttest, with a time interval of approximately a week.

\subsection{Design}

The experiment was a pretest-posttest design. In both pretest and posttest four interval AX (4IAX) discrimination and absolute identification with speech from one male speaker were administered. At the end of the posttest a short questionnaire was given.

On each trial of 4IAX discrimination, two different stimuli $A$ and $B$ were presented in one of eight possible orders: AB-AA, AA-BA, BA-AA, AA-AB, BB-AB, BB-BA, AB-BB or BA$\mathrm{BB}$. The listener had to indicate whether the first or the second pair consisted of the same stimuli. Both one-step and three-step discrimination were included. Learning by Acquired Distinctiveness would become most apparent in the one-step test, since the discrimination level in this test was low from the beginning. On the other hand, learning by Acquired Similarity would show in the three-step test, since its initially high discrimination levels could fall as a result of training. The 4IAX discrimination test was expected to reflect both phonemic and auditory perception of the stimulus pairs (Pisoni, \& Lazarus, 1974; Gerrits, 2001).

In absolute identification the listener has as many response options as there are stimuli. Since the listener is asked to indicate exactly which stimulus he heard, this test gives us insight into the listeners' control over the continuum.

For training, a classification design with trial-by-trial feedback on the correctness of the participants' responses was used. Classification was preferred over discrimination, since it directs the participants' attention towards the existence of two categories (e.g. Jamieson, \& Morosan, 1986). The training materials consisted of phoneme continua from five speakers, both males and females, other than the test speaker. Speaker variation was included to encourage robust category formation (Lively, Logan, \& Pisoni, 1993). 


\subsection{Procedure}

Listeners were tested individually. Half of them were told that they would hear English nonsense words, the other half of the participants were told that they would hear words from a foreign language. All experiments were run in a relatively quiet room at the Utrecht Institute of Linguistics OTS. A laptop computer was used both to present the stimuli at random and to register responses. Stimuli were presented over Beyerdynamic DT 770 headphones at a comfortable listening level.

The pretest was completed on the first day. On subsequent days, training sessions were run until the listener classified the new phoneme contrast correctly in at least $85 \%$ of the trials. On the final day the posttest took place. This test was of a similar content as the pretest, apart from a short questionnaire that was given after the listening tests. In this, the listeners were asked about the spelling of the newly learnt words. The following subsections will discuss the procedures of the listening tests in more detail.

\subsubsection{IAX Discrimination}

The first test administered during pretest and posttest was 4IAX discrimination. This test was given twice, once with one-step stimulus pairs (i.e. 1-2, 2-3, .., 7-8) and once with three-step stimulus pairs (i.e. 1-4, 2-5, .., 5-8). The order of these tests was balanced across subjects.

Listeners received written instructions in which they were asked to indicate whether the first or the second word pair they heard consisted of the same stimuli. It was stressed that differences could be small. Responses were given by striking one of two keys on the computer keyboard. A short task introduction was given, consisting of eight four-step stimulus pairs from the same continuum. Inter-stimulus intervals were set at $300 \mathrm{~ms}$, interpair intervals at $500 \mathrm{~ms}$, and response times were unlimited. The eight different orders per stimulus pair were each presented four times, resulting in 224 trials for the one-step and 160 trials in the three-step test. Trial order was randomized and there were three short breaks at regular intervals.

\subsubsection{Absolute Identification}

The second test in pre- and posttest was absolute identification. Listeners received written instructions, telling them to indicate exactly which stimulus they had heard from the continuum. The instructions included a picture of a row of eight buttons numbered ' 1 ' to ' 8 ' from left to right. Over the first and eighth buttons, pictures of a man wearing differently colored headphones were shown. It was explained that each button hid a unique word. The words changed in steps from the name of the first man (behind button 1) to the name of the second man (behind button 8). Next, the stimuli were introduced five times in increasing, decreasing and random sequences. Listeners were instructed to listen very carefully and to try to remember which button corresponded to which word. During testing, stimuli were presented 20 times in random order, resulting in a total of 160 trials. Listeners responded by mouse-clicking one of eight on-screen buttons. Response times were unlimited and there were two short breaks at regular intervals.

\subsubsection{Classification with feedback}

During training, a classification design was used. The categories were represented by the pictures of the men and were introduced as the men's names. Listeners had to reach a mean score of at least $85 \%$ correct identifications over two subsequent training tests before 
proceeding to the posttest. The test was introduced by the pronunciation of both endpoint stimuli by each of the five training speakers.

Listeners received immediate feedback on each trial, informing them of the correctness of their choice. The percentage of correct responses so far was shown regularly. Training tests each contained twelve repetitions per stimulus, resulting in 480 trials. There were three short breaks at regular intervals.

\section{Results}

Training results were represented as percentages of sif-responses per stimulus for each of the five speakers. Next, the phoneme boundary was determined at the 50\%-point for each of the five speakers in the training set and for each listener separately. Also, boundary widths were determined by calculating the $25 \%-75 \%$ range. Boundary widths reflect the steepness of the boundary. For 4IAX discrimination, percentages of correct responses were determined per stimulus. From absolute identification results, the mean responses to each of the stimuli and their variances were determined.

\subsection{Training}

The participants' results from the first and the last training session were compared with the English norm defined by 31 native listeners. The mean boundaries in the first and last training sessions differed significantly from those defined by the English listeners $[F(1,221)=7.9$, $p=.005$ and $F(1,222)=7.7, p=.006$, respectively]. Post-hoc analyses showed, however, that the mean boundary of only one speaker differed from the English norm in both training sessions. The Dutch listeners perceived more stimuli as /s/. In addition, the boundary value of one more speaker in the last training differed from that of the English listeners; again more stimuli were judged as $/ \mathrm{s} /$.

The results from the first training session did not lead to a full cross-over in $36 \%$ of the cases: usually, no $25 \%$-points were found, which means that listeners did not consistently choose the $/ \theta /$ category for stimuli at that end of the continuum. In the last training session, this number had decreased to only $6.3 \%$, approximately equal to the number of cases the natives missed $(6.5 \%)$. The boundary widths of the listeners for whom cross-overs were defined, differed significantly from the native English ones in the first training session $[F(1,186)=52.4, p<.001]$. In the last training session, however, these differences were no longer present.

\subsection{IAX Discrimination}

For one-step 4IAX discrimination, a repeated measures ANOVA was run with withinsubjects factors Test (pretest vs. posttest) and Stimulus Pair (1 through 7), and betweensubjects factors Listener Group (trained vs. control) and Language (English vs. foreign language). The results are shown in figure 2.

First of all, a main effect of Test was found, $F(1,30)=14.9, p=.001$. Listeners gave more correct answers in the posttest than in the pretest. Secondly, a main effect of Stimulus Pair was found, $F(6,180)=10.2, p<.001$. This means that listeners were not equally good at distinguishing all pairs of stimuli. No main effects of Listener Group or Language were found. The absence of these effects means that the test listeners did not perform considerably better than the controls, and that listeners in the English condition did not benefit from their knowledge of what language they were attending. A post-hoc ANOVA on the posttest data 
revealed only an effect of Listener Group, $F(1,224)=7.5, p=.007)$ : trained listeners scored better than those in the control group.
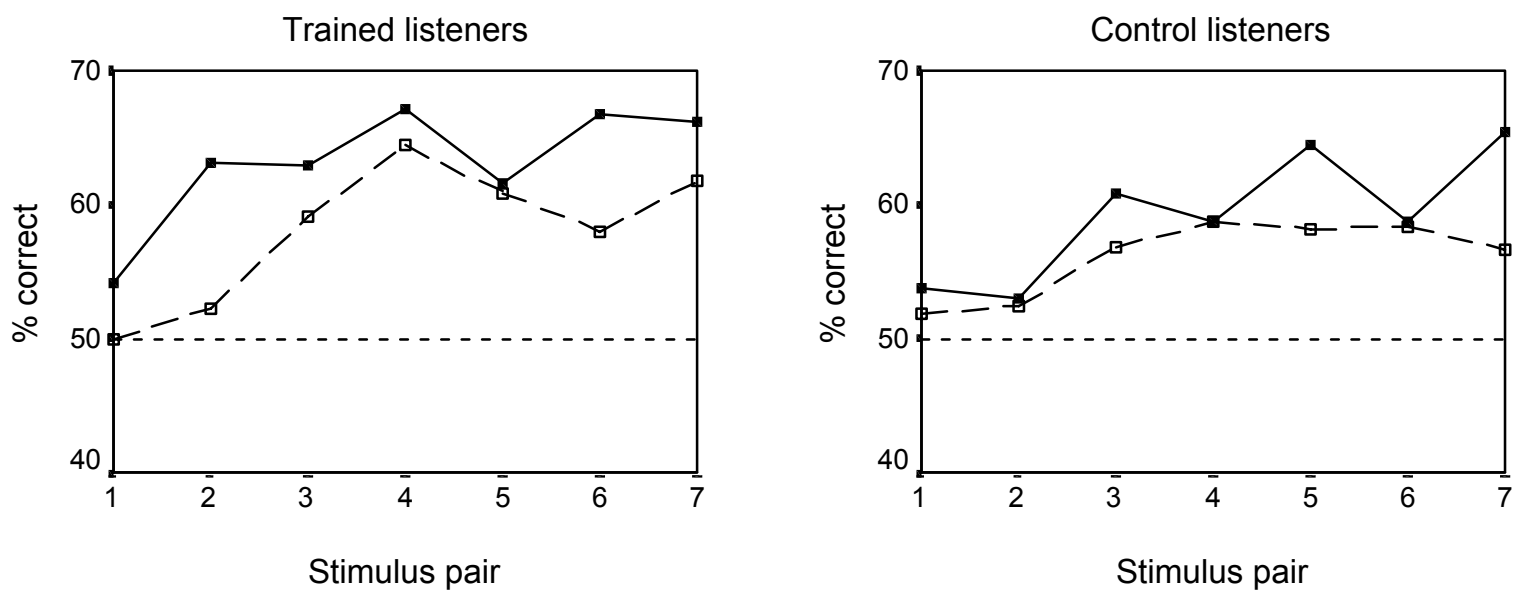

Figure 2. One-step 4IAX discrimination results for both pretest (dashed) and posttest (solid) with a reference line at chance level.

Three-step 4IAX discrimination showed similar results. Main effects of Test $[F(1,30)=13.4$, $p=.001)$ and Stimulus Pair $[F(3.4,101.4)=9.3, p<.001)$ were significant. Again, no main effect of Listener Group or Language was found. An ANOVA on the posttest data showed that test listeners performed better than controls, $F(1,155)=10.8, p=.001$.

\subsection{Absolute identification}

A repeated measures ANOVA was run on the mean responses shown in Figure 3 below, with within-subjects factors Test (pretest vs. posttest) and Stimulus (1 through 8) and betweensubjects factors Listener Group (trained vs. control) and Language (English vs. foreign language).

Firstly, a Test $\times$ Stimulus interaction $[F(4.5,136)=3.9, p=.003]$ was found. At the $/ \theta /$-end of the continuum, participants became better at identifying the stimuli, while this was not the case at the $/ \mathrm{s} /$-end. This can be seen in figure 3 by the trend towards the ideal case of absolute identifications at the $/ \theta /$-end only. Furthermore, main effects of Test $[F(1,30)=28.2, p<.001]$ and Stimulus $[F(2.6,78.3)=794.1, p<.001]$ were found. But again, no effects of the betweensubjects factors Listener Group and Language were present.

The mean variances showed main effects of Test $[F(1,30)=20.6, p<.001]$ and of Stimulus $[F(4.3,129.7)=5.5, p<.001)$. Posttest variances were smaller than those in the pretest, meaning that participants had become better at identifying the stimuli.

In summary, differences between pretest and posttest were present in the three tests. Trained listeners' progress, however, was hard to discriminate from that of control listeners. The fact that some test listeners needed only a few training sessions to reach criterion may partly account for this phenomenon, which will be investigated in subsection 3.4. 

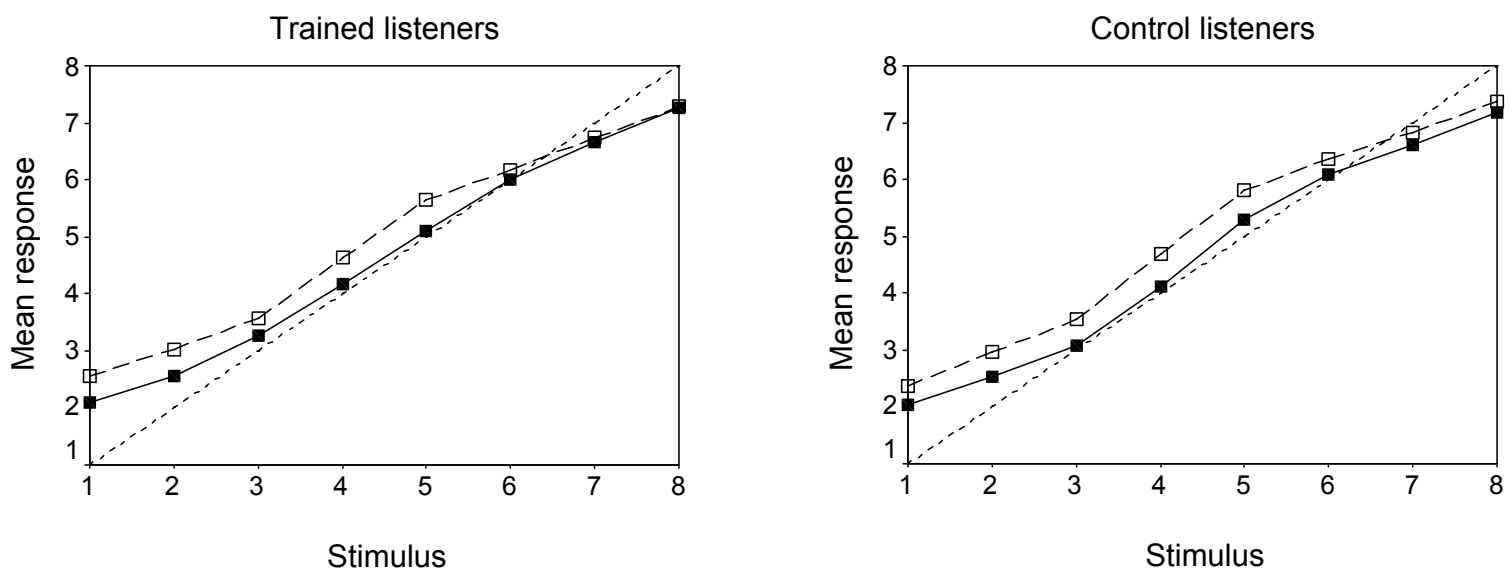

Figure 3. Absolute identification results for pretest (dashed) and posttest (solid). The ideal case of error-free perception is shown by the short-dashed line.

\subsection{The effect of amount of training on pretest-posttest differences}

The amount of training needed to reach criterion varied among test listeners: from 960 to 7,680 training trials before reaching criterion. It is conceivable that those listeners who needed more training also show larger differences between pre- and posttest results. Therefore, the trained listeners' data were tested in repeated measures ANOVAs, this time with Training Amount as a covariate. Only the findings that differ from the effects described in sections 3.2 and 3.3 will be reported here.

Three-step 4IAX discrimination showed a Test $\times$ Training Amount interaction $[F(1,15)=4.8$, $p=.044]$ as well as a main effect of Training Amount $[F(1,15)=16.4, p=.001]$. The rise in correct responses between pretest and posttest tended to be larger as the amount of training increased. The mean variances of the stimuli in absolute identification also showed a main effect of Training Amount, $F(1,15)=5.5, p=.033$.

\subsection{Posttest Questionnaire}

After the perception tests, listeners wrote down the words they had heard as the two extremes during absolute identification. The reported sets varied greatly. From the test group, six listeners reported the correct pair of words, but none of the control listeners succeeded in doing this. Remember that acoustically only the first consonant varied between stimuli thif and sif. Three types of errors were identified. Listeners (a) replaced target phonemes with other phonemes, (b) added phonemes that were not present in the signal or (c) reported hearing differences between the vowels or between the coda consonants.

In total, control listeners made more errors: 55 as opposed to 37 for the trained listeners. The controls made the majority of these, $49 \%$, in their responses to the first consonant, i.e. the target phoneme. They used almost twice as many substitutions, and also additions that resulted in complex onsets such as stif instead of sif. Furthermore, they more often replaced the coda consonant with another one, as in striss instead of thif.

\section{Discussion}

Listeners participated in training sessions until they learnt the contrast to the predetermined level of $85 \%$ correct. The training results showed that the phoneme boundaries were already close to the English norm during the first training session. But the widths of the phoneme 
boundaries became much smaller and even indistinguishable from the English norm as a result of training.

The pre- and posttest revealed main effects of Test for both discrimination and absolute identification tests. However, the absence of Test $\times$ Listener Group interactions showed that listeners - whether in the test or control group - all performed better when they did the tests for the second time. Differences between the test and control groups were only present within the posttest data of three-step discrimination and of the variance results in absolute identification. As for three-step discrimination, test listeners performed better than controls, especially at the $/ \theta /$-end of the continuum in the posttest. Response variances in absolute identification were smaller for the test group.

By taking into account the amount of training participants received, we found that listeners who needed many training sessions to reach the criterion of $85 \%$ correct responses, showed a larger difference between their pretest and posttest results for three-step discrimination and for the variances in absolute identification.

The main research question of this paper was: do Dutch adults learn the British-English $/ \theta-\mathrm{s} /$ contrast in a way compatible with Acquired Distinctiveness or with Acquired Similarity? We found no support for learning by Acquired Similarity. For this to be the case, perceptual sensitivity to speech sounds that belong to the same category should decrease, but we found no such effect at all. On the contrary, the improvement we found for the discrimination tests mainly occurred within instead of between categories. So these findings do not strongly support our other hypothesis, Acquired Distinctiveness, either.

Despite the progress during training, little evidence of an increase in discrimination levels at the phoneme boundary was found, contrary to earlier findings (Jamieson, \& Morosan, 1986). This may have been caused either by the nature of the tasks used in pretest and posttest, or by the participants' high pretest levels. Firstly, the tasks used may have directed the listeners' attention too much towards the acoustic differences between the stimuli by testing auditory instead of phonemic perception. However, we expected to find a combination of these listening levels in our results (Gerrits, 2001; Pisoni, \& Lazarus, 1974). Secondly, most participants performed already quite well in the pretest (see, for example, figure 2). The room left for improvement as a result of training was thereby restricted and may have made such improvement difficult to distinguish from improvement by task repetition. We also think that the pretest was a training in itself due to its length, which helped control listeners to improve their scores in the posttest. Most earlier studies, however, did not involve a control group (e.g. Strange, \& Dittmann, 1984; Logan, Lively, \& Pisoni, 1991) and could therefore only report the test group's progress.

An explanation that may account for a portion of the errors made by the participants in reporting which words they had heard, is an effect similar to 'verbal transformation' (Warren, 1961). In Warren's study, listeners heard uninterrupted repetitions of a word or phrase. They reported hearing words that were not present in the speech signal. In our study, comparable misperceptions were reported. Even though there were silent intervals between subsequent presentations of word forms, perceptual disturbances may still have occurred. Schouten \& van Hessen (1998), for example, reported that their participants 'hallucinated' as a result of prolonged exposure to speech sounds taken from a phoneme continuum. The fact that control listeners made more errors than trained listeners may be explained by the differences in speaker variation the two listener groups had been exposed to. During the course of the experiment, trained listeners heard the word forms spoken by six different voices, whereas control listeners heard only the test speaker's voice. 
A sub-question that was addressed in the present study was whether the availability of knowledge of the language you are learning influences perceptual learning. We found that participants who knew they were listening to English, did not benefit from this knowledge. So either listeners in both the English and the Foreign Language conditions used their knowledge of English equally, or neither of the groups accessed this knowledge.

\section{Conclusion}

Dutch listeners improved their perception of British-English $/ \theta$-s/ during training. Trained listeners performed better in the posttest than in the pretest and in several respects they also did better than the control group. Their improved performance excluded Acquired Similarity, but did not strongly support Acquired Distinctiveness either. This lack of effect was thought to be due to both the high pretest performances of our participants and the nature of the tests used in pretest and posttest. Furthermore, control listeners, who received no training, also improved by simply performing the tests in pretest and posttest twice. These results show that it is important to include a control group into the design of a phoneme training study, which has often not been the case. Finally, listeners who knew that they were listening to BritishEnglish did not benefit from this knowledge opposed to listeners who were told they were listening to a foreign language.

\section{References}

Best, C.T., Traill, A., Carter, A., Harrison, K.D. \& Faber, A. (2003). !Xóõ click perception by English, Isizulu, and Sesotho listeners. In M. J. Solé, D. Recasens \& J. Romero (Eds.), Proceedings of the 15th International Congress of Phonetic Sciences; Barcelona, 3-9 Augustus 2003 (pp. 853-856).

Collins, B.S. \& Mees, I.M. (1999). The phonetics of English and Dutch. Leiden: Brill.

Ganong, W.F. (1980). Phonetic categorization in auditory word perception. Journal of Experimental Phsychology: Human Perception and Performance, 6, 110-125.

Gerrits, E. (2001). The categorisation of speech sounds by adults and children. Doctoral Dissertation, Utrecht University.

Hessen, A.J. van (1992). Discrimination of familiar and unfamiliar speech sounds. Doctoral Dissertation, Utrecht University.

Jamieson, D.G. \& Morosan, D.E. (1986). Training non-native speech contrasts in adults: acquisition of the English /ð/-/0/ contrast by francophones. Perception \& Psychophysics, 40, 205-215.

Logan, J.S., Lively, S.E., \& Pisoni, D.B. (1991). Training Japanese listeners to identify English /r/ and /1/: A first report. Journal of the Acoustical Society of America, 89, 874-886.

Liberman, A.M., Harris, K.S., Kinney, J.A. \& Lane, H. (1961) The discrimination of relative onset-time of the components of certain speech and nonspeech patterns, Journal of Experimental Psychology, 61, 379388.

Lively, S.E., Logan, J.S., \& Pisoni, D.B. (1993). Training Japanese listeners to identify English /r/ and /1/. II The role of phonetic environment and talker variability in learning new perceptual categories. Journal of the Acoustical Society of America, 94, 1242-1255.

Pisoni, D.B., and Lazarus, J.H. (1974). Categorical and noncategorical modes of speech perception along the voicing continuum. Journal of the Acoustical Society of America, 55, 328-333.

Pisoni, D.B. (1991). Modes of processing speech and nonspeech signals. In I.G. Mattingly \& M. StuddertKennedy (Eds.): Modularity and the motor theory of speech perception (pp. 225-238). Hillsdale NJ: Lawrence Erlbaum Associates.

Schouten, M.E.H., \& Hessen, A.J. van (1998). Response distributions in intensity resolution and speech discrimination. Journal of the Acoustical Society of America, 104, 2980-2990.

Strange, W. \& Dittmann, S. (1984). Effects of discrimination training on the perception of /r-1/ by Japanese adults learning English. Perception \& Psychophysics, 36, 131-145.

Strange, W. \& Jenkins, J.J. (1978). Role of linguistic experience in the perception of speech. In R.D. Walk \& H.L. Pick (Eds.): Perception and experience (pp. 125-169). New York: Plenum Press.

Warren, R.M. (1961). Illusory changes of distinct speech upon repetition - the verbal transformation effect. British Journal of Psychology, 52, 249-258. 
Werker, J.F. \& Tees, R.C. (1984). Cross-language speech perception: evidence for perceptual reorganization during the first year of life. Infant Behavior and Development, 7, 49-63. 